\title{
SEM imaging of acoustically stimulated charge transport in solids
}

Cite as: Appl. Phys. Lett. 110, 264103 (2017); https://doi.org/10.1063/1.4986779

Submitted: 21 March 2017 . Accepted: 06 June 2017 . Published Online: 29 June 2017

Evgeny Emelin, H. D. Cho (D), Zeke Insepov (D), J. C. Lee (D), Tae Won Kang, Gennady Panin, Dmitry Roshchupkin, and Kurbangali Tynyshtykbayev (D)

ARTICLES YOU MAY BE INTERESTED IN

Modulation of acousto-electric current using a hybrid on-chip AIN SAW/GFET device Applied Physics Letters 110, 243504 (2017); https://doi.org/10.1063/1.4986481

Unexpected significant increase in bulk conductivity of a dielectric arising from charge injection

Applied Physics Letters 110, 262902 (2017); https://doi.org/10.1063/1.4990677

Nanometer size hole fabrication in $2 \mathrm{~d}$ ultrathin films with cluster ion beams

AIP Advances 7, 075014 (2017); https://doi.org/10.1063/1.4996185

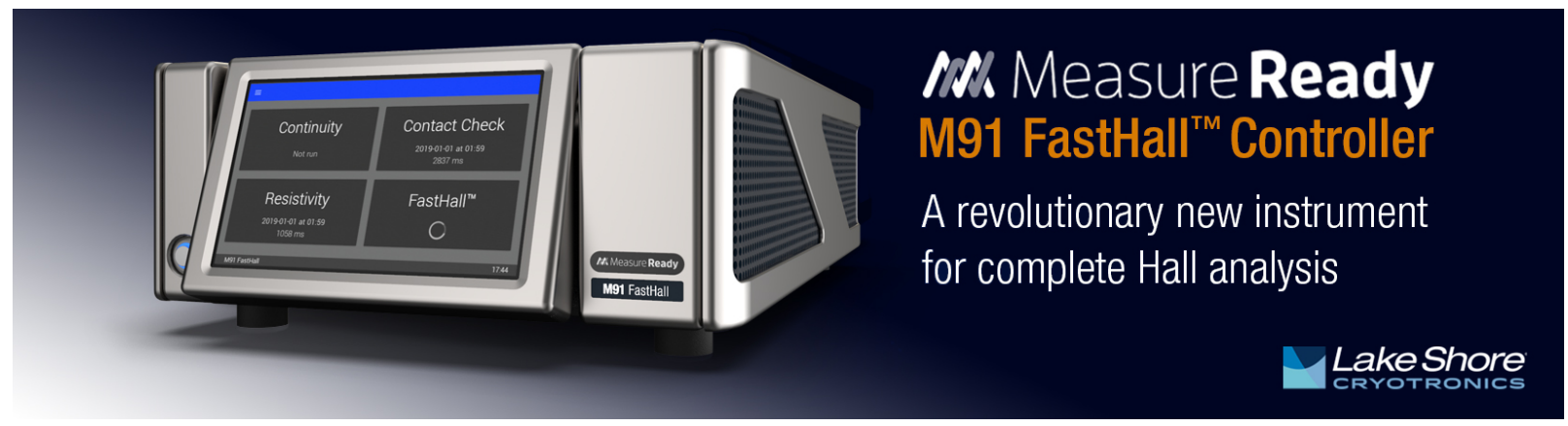




\title{
SEM imaging of acoustically stimulated charge transport in solids
}

\author{
Evgeny Emelin, ${ }^{1}$ H. D. Cho, ${ }^{2}$ Zeke Insepov, ${ }^{3,4,5}$ J. C. Lee, ${ }^{2}$ Tae Won Kang, ${ }^{2}$ \\ Gennady Panin, ${ }^{1,2}$ Dmitry Roshchupkin, ${ }^{1,6}$ and Kurbangali Tynyshtykbayev ${ }^{3}$ \\ ${ }^{1}$ Institute of Microelectronics Technology and High-Purity Materials Russian Academy of Sciences, \\ Chernogolovka 142432, Russia \\ ${ }^{2}$ Quantum-Functional Semiconductor Research Center, Dongguk University, 3-ga 26 Pildong Junggu \\ Seoul 100-715, Korea \\ ${ }^{3}$ Nazarbayev University Research and Innovation System, 53 Kabanbay Batyr St., Astana 010000, Kazakhstan \\ ${ }^{4}$ National Research Nuclear University MEPhI, Kashirskoye sh., 31, Moscow, 115409, Russia \\ ${ }^{5}$ Purdue University, School of Nuclear Engineering, 400 Central Drive, West Lafayette, Indiana 47907-2017, \\ USA \\ ${ }^{6}$ National University of Science and Technology MISiS, Leninskiy prospekt 2, 119049 Moscow, Russia
}

(Received 21 March 2017; accepted 6 June 2017; published online 29 June 2017)

\begin{abstract}
Acoustically stimulated charge transport in solids was studied using the scanning electron microscopy method (SEM). The surface acoustic wave on the surface of the YZ-cut of a $\mathrm{LiNbO}_{3}$ crystal was visualized by registration of low-energy secondary electrons in SEM, and the charge distribution on the crystal surface was visualized using the electron beam induced current method. To register the induced current, an interdigital transducer structure was formed from graphene on the crystal surface. It was shown that the charge distribution on the crystal surface corresponds to the distribution of the acoustic wave field on the crystal surface. Published by AIP Publishing.

[http://dx.doi.org/10.1063/1.4986779]
\end{abstract}

Applications of surface acoustic waves (SAWs) are of great interest for solar energy for the acoustically stimulated transport of charge carriers generated in semiconductors and dielectrics under the influence of light. ${ }^{1-4}$ A prospective application of SAWs in solar cells could provide a $90 \%$ increase in the cell efficiency. SAWs propagating in piezoelectric crystals (piezoelectric semiconductor GaN and GaAs crystals included) have opposite potential values in the SAW minima and maxima due to the piezoelectric effect. Electrons and holes generated by light in a semiconductor or in the subsurface layer of a piezoelectric crystal are correspondingly distributed between SAW minima and maxima. The charges are then transported by SAWs to the solar cell exit at the acoustic wave velocity. Taking advantage of the SAW presence in solar cells, the area of charge "harvest" from the surface of a semiconductor structure or a piezoelectric crystal can be increased, and correspondingly, the solar cell efficiency can be increased too.

Perspectives in solar energy are associated with the ability to use graphene, which can be used as a structure in which the electron-hole pairs are generated under the influence of radiation. ${ }^{5,6}$ Moreover, a graphene film is a good medium for SAW propagation. The possibility of charge transport by SAWs in graphene and the possibility of monitoring a SAW under an electric potential applied to graphene were reported in a number of works. ${ }^{7-14}$

The SEM method can be used to study the SAW propagation in piezoelectric crystals. This method enables the visualization of acoustic wave propagation through registration of low-energy secondary electrons sensitive to electric fields accompanying the SAW propagation in piezoelectric crystals (the electric fields between SAW minima and maxima). ${ }^{15-19}$ In this case, the acoustic wave field on the crystal surface can be visualized, acoustic wavelengths and power flow angles can be measured, and the interaction of acoustic waves with the crystal lattice defects can be studied.

The aim of this work was to study the charge transport by SAWs. To visualize the charge transport, the electron beam induced current method (EBIC) method was used, which permits the visualization of charge distribution on the crystal surface by measuring the current flowing through the sample. ${ }^{20}$ Charges introduced by the primary electron beam and those generated in the crystal subsurface area are distributed between the SAW minima and maxima according to the potential sign and are then carried by SAWs at the acoustic wave velocity towards the current collector at the exit.

The YZ-cut of a $\mathrm{LiNbO}_{3}$ crystal was used to visualize the acoustically stimulated charge transport. Figure 1 shows a SEM micrograph of the SAW device used to study the charge transport on the crystal surface. The SAW with a wavelength of $\Lambda=60 \mu \mathrm{m}$ was excited using an Al interdigital transducer (IDT) structure with a period of $15 \mu \mathrm{m}$ formed on the crystal surface using the photolithography method. The IDT at the resonance excitation frequency of $f=58.13 \mathrm{MHz}$ excites a SAW with the wavelength of $\Lambda=60$ which propagates in the $Y Z$-cut of the $\mathrm{LiNbO}_{3}$ crystal along the $Z$ axis at the velocity of $V=\Lambda \times f=3488 \mathrm{~m} / \mathrm{s}$. To register the charge transport with the SAW, a graphene IDT (current collector) was formed on the $\mathrm{LiNbO}_{3}$ crystal surface. The graphene film was formed on the crystal surface using the transfer technique. The initial graphene film was grown on the surface of a Ni film using the CVD method. ${ }^{21}$

The graphene IDT structure (Fig. 1) was fabricated using the photolithography method and subsequent plasmachemical etching of the structure in the oxygen plasma. The period of the graphene IDT structure is $60 \mu \mathrm{m}$.

SAW propagation in the $Y Z$-cut of a $\mathrm{LiNbO}_{3}$ crystal was visualized using a SEM at the accelerating voltage of $E=1 \mathrm{kV}$ and the electron probe current of $I=1$ na. A higher accelerating 
(a)
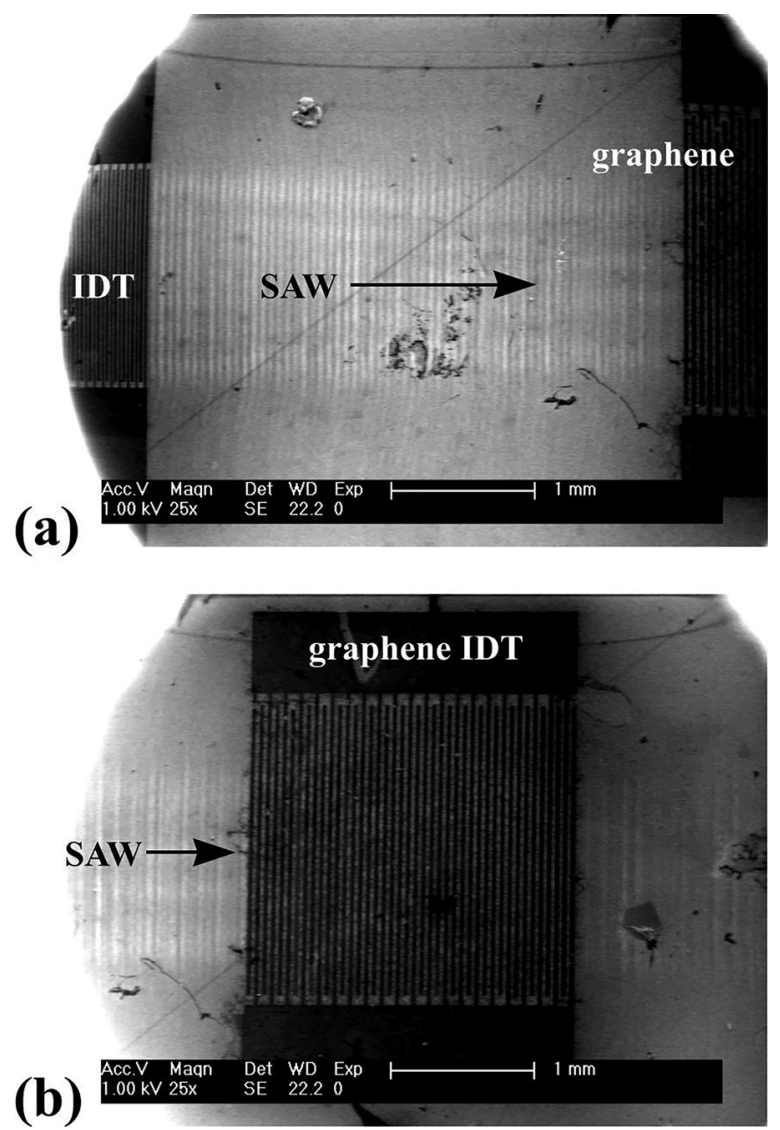

FIG. 1. SAW device: (a) SEM image of the SAW and (b) graphene current collector. The YZ-cut of a $\mathrm{LiNbO}_{3}$ crystal is shown: $\Lambda=60 \mu \mathrm{m}$ and $f=58.13 \mathrm{MHz}$.

voltage is impermissible because at higher voltages, the crystal surface becomes negatively charged and the image becomes strongly distorted. Figure 1(a) shows an image of the SAW excited by the IDT at the resonance excitation frequency of $f=58.13 \mathrm{MHz}$ and propagating to the graphene current collector (graphene IDT). Figure 1(b) displays a micrograph of the graphene current collector and an image of the acoustic wave field near it.

Charge distribution on the surface of the YZ-cut of the $\mathrm{LiNbO}_{3}$ crystal was simultaneously visualized using the EBIC method. An EBIC image of the crystal surface was obtained by measuring the current on the graphene collector during the process of electron beam scanning using SEM. Figure 2 presents an image of the crystal surface obtained using the EBIC method. Figure 2(a) shows a periodic structure corresponding to the charge distribution on the crystal surface. The gap width of IDT is $30 \mu \mathrm{m}$, which corresponds to the SAW wavelength of $\Lambda=60 \mu \mathrm{m}$, i.e., the charges are distributed between the minima and maxima of the surface acoustic wave. A light contrast corresponds to positive charges, while the dark contrast corresponds to negative charges. In the area of the graphene current collector, current is distributed in accordance with the structure period. The gap $d$ between the IDT fingers in this area is $60 \mu \mathrm{m}$, which is also a period of the graphene IDT. Figure 2(b) displays the distribution of the EBIC contrast on the crystal surface along the $Z$ axis. A periodic modulation of the EBIC contrast with the period of $\Lambda=2 d=60 \mu \mathrm{m}$ can be seen on the free crystal surface, which corresponds to the distribution of positive and negative
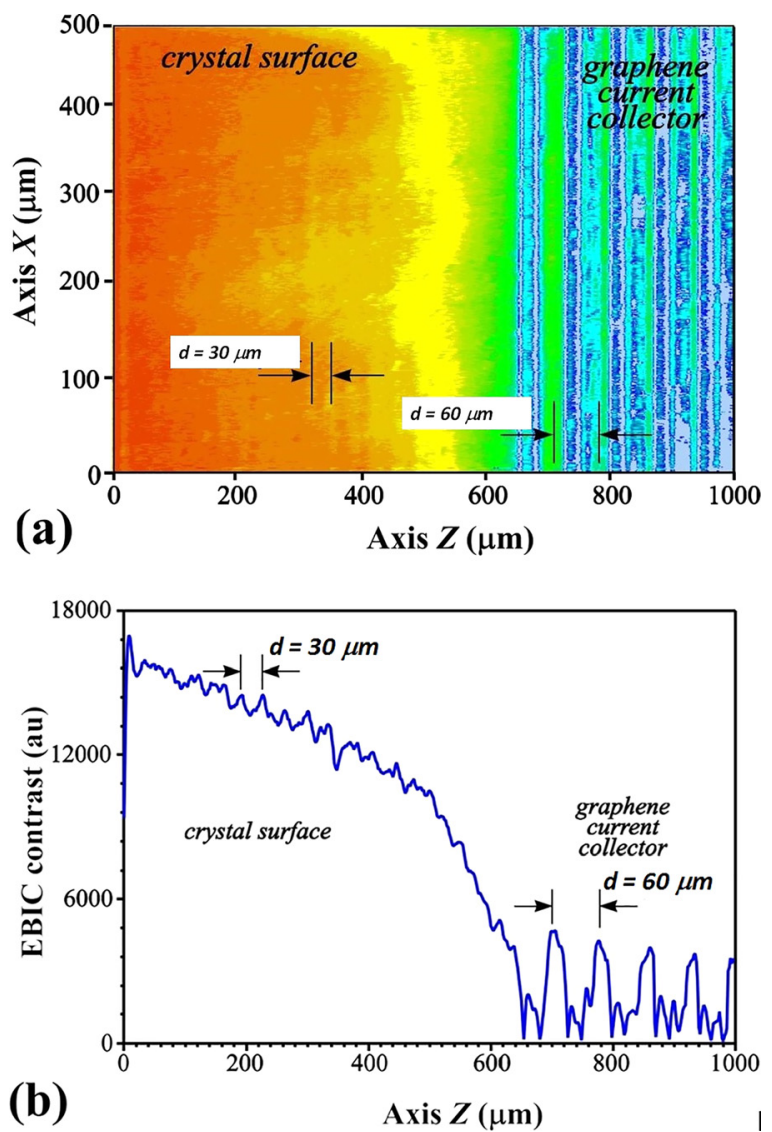

FIG. 2. EBIC image of the charge distribution on the crystal surface: (a) 2D map and (b) EBIC contrast of the charge distribution.

charges between the SAW minima and maxima. In the area of the graphene current collector, a periodic structure with the period of $120 \mu \mathrm{m}$ and of a very high contrast (ratio of minima and maxima) is observed, which conforms to the graphene IDT registration of charges delivered by the SAW.

Thus, this work reports the visualization of the distribution of charges, which were introduced into a dielectric substrate by a primary electron beam and those generated during the interaction of the beam with the crystal lattice, between the SAW minima and maxima using the EBIC method. The transport of charge carriers by the surface acoustic wave to the graphene collector is also presented.

The proposed method is significantly simpler and more reliable compared to the electrostatic force microscopy (EFM) method, ${ }^{22,23}$ in a sense that the latter method is not suitable for imaging dynamic surface pictures of the fast moving surface acoustic waves.

This work was supported by the Russian Foundation for Basic Research (Grants No. 16-52-51041 and No. 16-2906306) and NUST "MISiS" (Grant No. K1-2015-046) and in part by the Nazarbayev University World Science Stars program (Grant No. 031-2013 of 12/3/2013).

${ }^{1}$ C. Rocke, S. Zimmermann, A. Wixforth, J. P. Kptthaus, G. Böhm, and G. Weimann, Phys. Rev. Lett. 78, 4099 (1997).

${ }^{2}$ P. D. Batista, R. Hey, and P. V. Santos, Appl. Phys. Lett. 92, 262108 (2008).

${ }^{3}$ S. J. Jiao, P. D. Batista, K. Biermann, R. Hey, and P. V. Santos, J. Appl. Phys. 106, 053708 (2009). 
${ }^{4}$ V. M. Yakovenko, Physica B 407, 1969 (2012).

${ }^{5}$ K. S. Novoselov, A. K. Geim, S. V. Morozov, D. Jiang, M. I. Katsnelson, I. V. Grigorieva, S. V. Dubonos, and A. A. Firsov, Nature 438, 197 (2005).

${ }^{6}$ M. I. Katsnelson and K. S. Novoselov, Solid State Commun. 143, 3 (2007).

${ }^{7}$ V. Miseikis, J. E. Cunningham, K. Saeed, R. O'Rorke, and A. G. Davies, Appl. Phys. Lett. 100, 133105 (2012).

${ }^{8}$ O. A. C. Nunes, J. Appl. Phys. 115, 233715 (2014).

${ }^{9}$ S. Zheng, H. Zhang, Z. Feng, Y. Yu, R. Zhang, C. Sun, J. Liu, X. Duan, W. Pang, and D. Zhang, Appl. Phys. Lett. 109, 183110 (2016).

${ }^{10}$ P. V. Santos, T. Schumann, M. H. Oliveira, Jr., J. M. J. Lopes, and H. Riechert, Appl. Phys. Lett. 102, 221907 (2013).

${ }^{11}$ T. Poole, L. Banedhu, and G. R. Nash, Appl. Phys. Lett. 106, 133107 (2015).

${ }^{12}$ Y. Chen, H. Zhang, H. Zhang, Z. Fang, H. Zhao, C. Sun, S. Zheng, W. Pang, and D. Zhang, Appl. Phys. Lett. 108, 033107 (2016).

${ }^{13}$ D. Roshchupkin, L. Ortega, I. Zizak, O. Plotitcyna, V. Matveev, O. Kononenko, E. Emelin, A. Erko, K. Tynyshtykbayev, D. Irzhak, and Z. Insepov, J. Appl. Phys. 118, 104901 (2015).
${ }^{14}$ Z. Insepov, E. Emelin, O. Kononenko, D. V. Roshchupkin, K. B. Tynyshtykbayev, and K. A. Baigarin, Appl. Phys. Lett. 106, 023505 (2015).

${ }^{15}$ W. J. Tanski and D. Wittels, Appl. Phys. Lett. 34, 537 (1979).

${ }^{16}$ G. Eberharter and H. P. Feuerbaum, Appl. Phys. Lett. 37, 698 (1980).

${ }^{17}$ H. Bahadur and R. Parshad, Scanning Electron Microsc. 1, 509 (1980).

${ }^{18}$ D. V. Roshchupkin and M. Brunel, IEEE Trans. Ultrason. Ferroelectr. Freq. Control 41, 512 (1994).

${ }^{19}$ D. V. Roshchupkin, M. Brunel, R. Tucoulou, E. Bigler, and N. G. Sorokin, Appl. Phys. Lett. 64, 164 (1994).

${ }^{20}$ H. J. Leamy, J. Appl. Phys. 53, R51 (1982).

${ }^{21}$ O. V. Kononenko, V. N. Matveev, D. P. Field, D. V. Matveev, S. I. Bozhko, D. V. Roshchupkin, E. E. Vdovin, and A. N. Baranov, Nanosyst.: Phys., Chem., Math. 5(1), 117 (2014).

${ }^{22}$ G. H. Buh, H. J. Chung, and Y. Kuk, Appl. Phys. Lett. 79, 2010 (2001).

${ }^{23}$ N. S. Malvankar, S. E. Yalcin, M. T. Tuominen, and D. R. Lovley, Nat. Nanotechnol. 9, 1012-1017 (2014). 